\title{
Cumulative Binomial Distribution Computer
}

SANDIA CORPORATION

ALBUQUERQUE, NEW MEXICO



RELIABILITY DEPARTMENT

\section{UNIVERSTTY OF} ARIZONA LIBRARY

metadc 303860

MN 341965 
SC-R-64-1348

Sandia Corporation

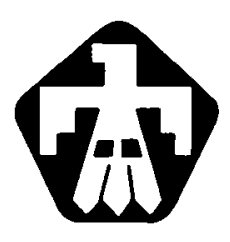

$\bullet \bullet \bullet \bullet \bullet \bullet \bullet$ MONOGRAPH

THE CUMULATIVE BINOMIAL DISTRIBUTION COMPUTER

J. O. Muench

October 1964 
Published by

Sandia Corporation,

a prime contractor to the

United States Atomic Energy Commission

LEOA NOTICE

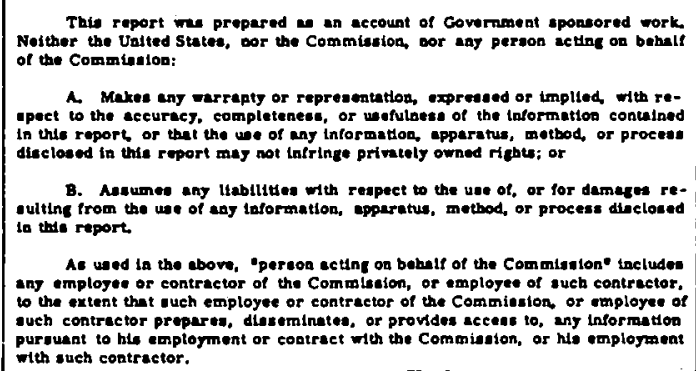

Printed in USA. Price \$1.50. Available from the Clearinghouse for Federal Scientific and Technical Information, National Bureau of Standards,

U. S. Department of Commerce, Springfield, Virginia 
THE CUMULATIVE BINOMIAL DISTRIBUTION COMPUTER

by

J. O. Muench

October 1964 


\section{Acknowledgment}

The Cumulative Binomial Distribution Computer incorporates valuable suggestions made by supervision and members of the Sandia Corporation Reliability Department. 
THE CUMULATIVE BINOMIAL DISTRIBUTION COMPUTER equation

The Cumulative Binomial Distribution Computer is designed to provide an approximate solution to the

\section{where}

$$
F(c)=\sum_{x=0}^{c}\left(\begin{array}{l}
n \\
x
\end{array}\right) p^{x}(1-p)^{n-x}
$$

$F(c)=$ the probability of observing $c$ or less units with a specified characteristic

$\mathbf{n}=$ the number of observations

$c=$ the number of units or events with specified characteristic

$p=$ the fraction of the population with the specified characteristic 
The physical layout of the Cumulative Binomial Distribution Computer was designed around the above form of the cumulative binomial equation so that operating characteristic curves for single sampling plans could be evaluated with minimum effort by the user. The solution to other forms of the cumulative binomial equation can be obtained from the computer but somewhat less directly. The Cumulative Binomial Distribution Computer will provide the solutions to more problems than those solved by an earlier work of the author--A Confidence Limit Computer (Reference 1). The increased versatility of the Cumulative Binomial Distribution Computer makes it a recommended replacement for the Confidence Limit Computer.

The following examples illustrate typical problems the computer will solve. The examples were not chosen to enlighten the reader in probability or sampling theory, but rather to demonstrate some practical applications of the computer. The binomial distribution is discussed in the preface of The Tables of the Cumulative Binomial Probability Distribution (Reference 2).

In using the computer there is no "correct" or "incorrect" side. If you can obtain a reading you are using the "correct" side. If you cannot obtain a reading on one side then try the other side. If you still cannot get a reading, the value is off scale. 
The Cumulative Binomial Distribution Computer can be used to solve problems of the general type: What is the probability, $F(c)$, that an event with probability $p$ occurs
a. c or fewer times in $\mathrm{n}$ trials?
b. more than $\mathrm{c}$ times in $\mathrm{n}$ trials?
c. c or more times in $n$ trials?
d. exactly $c$ times in $n$ trials?

\section{Example 1.}

An event has a probability $p$ of 0.01 . In 400 trials what is the probability of observing
a. 8 or less?
b. more than 8 ?
c. 8 or more?
d. exactly 8 ? 
Align $n=400$ with $p=0.01$. This setting of the computer will provide the solution to all four parts of the problem so do not move the slide.

\section{Solution:}

a. Where the red curve $c=8$ crosses the diagonal black scale, read 0.98 . This is the probability the event will occur eight times or less.

b. All we need here is the complement of a., above. $1-0.98=0.02$. This is the probability the event will occur more than eight times.

c. To determine the probability of the event occurring eight or more times, we must determine the probability of seven or less occurrences and subtract from one. Where the red curve $c=7$ crosses the diagonal black scale, read $0.95 .1-0.95=0.05$. This is the probability of the event occurring eight or more times. 
d. For the probability of exactly eight occurrences we must first determine the probability of eight or less occurrences, then determine the probability of seven or less occurrences. The difference will be the probability of exactly eight occurrences. In a., above, we determined the probability of eight or less occurrences to be 0.98 . In c., above, we determined the probability of seven or less occurrences to be 0.95 . Therefore, the probability of exactly eight occurrences is $0.98-0.95=0.03$.

\section{Example 2. (Unknown Fraction Defective, p)}

Plot the operating characteristic (OC) curve (probability of acceptance versus fraction defective) of the following sampling plan:

Sample size $\mathrm{n}=50 \quad$ Acceptance number $\mathrm{c}=2$.

To determine points on the OC curve select the side of the computer with an $\mathrm{n}$ scale from 2 to 100. Set the red curve $c=2$ on 0.999 (an arbitrarily chosen probability of acceptance) on the diagonal black scale. Read on the $n$ scale underneath 50 a $p$ value (fraction defective) of approximately 0.0038. The values 0.999 and 0.0038 determine the location of one point on the OC curve. Move the computer slide to the left until the red curve $c=2$ rests on 0.95 on the diagonal black scale. Underneath an $n$ of 50 read a value of 0.016 . The values 0.95 and 0.016 determine the location of another point on the OC curve. Move the slide to the 
left and set the curve for $c=2$ at 0.90 and obtain a $p$ value. Continue to obtain readings for $c=2$ along the entire length of the black diagonal scale as frequently as desired, depending upon the degree of definition desired in the OC curve. An example of one set of readings for plotting the subject OC curve is as follows:

Probability of 0,1 , or 2 Defectives in Sample. The Probability of Acceptance. $P_{\mathbf{a}}$. (Set Red Curve

$c=2$ to These Values.)
0.999
0.95
0.90
0.80
0.70
0.60
0.50
0.40
0. 30
0.20
0. 10
0.05
0.001

Fraction Defective in Lot. p.

(Read These Values Under an $n$ of 50 on the Computer.)
0.0038
0. 016
0. 022
0.031
0.038
0.045
0.054
0. 061
0. 071
0.084
0. 10
0. 14
0. 19 
Plotting the values gives:

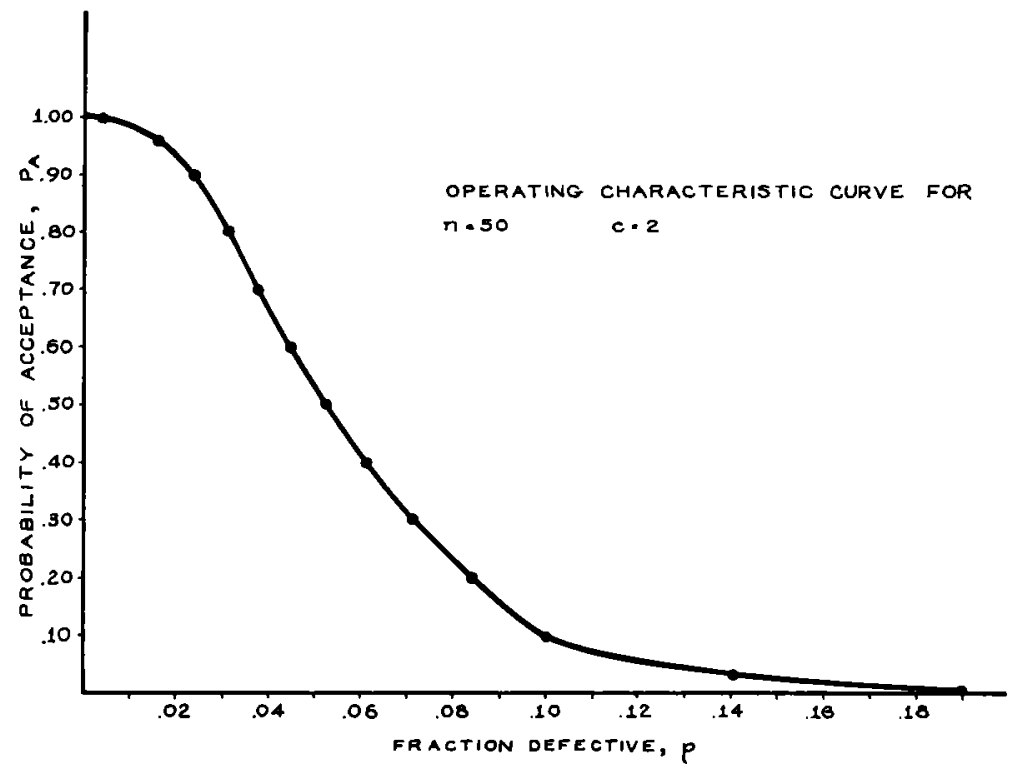


Example 3. (Unknown Probability of Acceptance, $P_{a}$, and Unknown Probability of Rejection, $P_{r}$ )

The sample size is 10 . No defectives are permitted in the sample if the lot is to be accepted. The fraction defective is 0.045 . (a) What is the probability of acceptance? (b) What is the probability of rejection?

a. To determine the $P_{a}$ locate the $n$ value of 10 . Align a $p$ value of 0.045 with the $n$ value of 10. On the red curve $c=0$ where the curve crosses the diagonal black line, read a $P_{a}$ of approximately 0.64 .

b. $\quad P_{r}=1-P_{a}=1-0.64=0.36$.

Example 4. (Unknown Sample Size, n)

It is desired to accept 95 percent of the lots of material containing a fraction defective no greater than 0.001 . What should the sample size be?

Note that no acceptance number is specified, yet the sample size depends upon the acceptance number. Therefore, we will arbitrarily start with an acceptance number of zero and determine the sample size. After that we will proceed to acceptance numbers $1,2,3$, etc., and determine the corresponding sample size. 
Using the appropriate side of the computer, align the red curve $c=0$ with 0.95 on the diagonal black scale. Read above a $p$ value of 0.001 an $n$ value of 49 . Therefore, the sample size is 49 for an acceptance number of zero. Next, move the slide to the left until the red curve $c=1$ aligns with 0.95 on the diagonal black scale. Locate a $p$ value of 0.001 and you will find a blank space above it. Turn the computer over and align the red curve $c=1$ with 0.95 on the diagonal black scale. Above a $p$ value of 0.001 read an $n$ value of 360 . When the acceptance number is one, the sample size is 360 . The procedure for determining the sample size for larger acceptance numbers is the same as just described. Check your readings against the following:

\section{Acceptance Number, c}

0

1

2

3

4

5

10

50

100

\section{Sample Size, n}

360

825

1,400

2,025

2,700

6,250

40,000

87,500 


\section{Example 5. (Unknown Acceptance Number, c)}

The sample size is 50 . The fraction defective is 0.10 . (a) What acceptance number, $c$, should be used for a probability of acceptance, $P_{a}$, of 0.95 ? (b) $A P_{a}$ of 0.10 ?

a. Locate an $\mathrm{n}$ of 50. Align with the $50 \mathrm{a} \mathrm{p}$ value of 0.1 . On the diagonal black scale at 0.95 read a $c$ value of 8 . The acceptance number is 8 for a $P_{a}$ of 0.95 .

b. Do not move the slide. On the diagonal black scale at 0.10 read a c value of 2 . The acceptance number is 2 for a $P_{a}$ of 0.10 .

Note that without moving the slide an acceptance number, $c$, can be obtained for various probabilities of acceptance, $P_{a}$.

\section{The Upper One-Sided Confidence Limit}

The usual equation for an upper binomial confidence limit is:

$$
1-\gamma=\sum_{j=0}^{c}\left(\begin{array}{l}
n \\
j
\end{array}\right) p^{j}(1-p)^{n-j}
$$


where
$\gamma=$ the confidence level
$\mathbf{n}=$ the number of observations
$c=$ the (observed) number of units or events with specified characteristic
$\mathrm{p}=$ the upper confidence limit.

To use the Cumulative Binomial Distribution Computer take $F(c)=1-\gamma$. That is, if it is desired to use a confidence level of 0.90 , subtract the 0.90 from one. The remainder, 0.10 , is $F(c)$ which is read on the diagonal black scale. For a 0.99 confidence level read 0.01 on the diagonal black scale, etc.

\section{Example 6.}

If a sample of 150 items is tested (or inspected) and there are four failures (or defectives), what is the upper confidence limit at the 0.95 confidence level? 
Use the side of the computer with an $n$ range of 100 to 100,000 . Locate the red curve $c=4$. Set this curve at 0.05 on the diagonal black scale $(0.05=1-0.95)$. Read under $n=150$ the upper confidence limit approximately 0.06 ; in other words, with 95 -percent confidence the true fraction defective is not greater than approximately 0.06 .

\section{The Lower One-Sided Confidence Limit}

The usual equation for a lower one-sided confidence limit is

$$
1-\gamma=\sum_{j=d}^{n}\left(\begin{array}{l}
n \\
j
\end{array}\right) p^{j}(1-p)^{n-j}
$$

where

$\gamma=$ the confidence level

$\mathrm{n}=$ the number of observations

$d=$ the (observed) number of units or events with specified characteristic

$\mathrm{p}=$ the lower confidence limit. 
To use the Cumulative Binomial Distribution Computer solve the equation

$$
F(c)=\sum_{j=0}^{c}\left(\begin{array}{l}
n \\
j
\end{array}\right) p^{j}(1-p)^{n-j}
$$

where $c=d-1$ and $F(c)=\gamma=$ the confidence level.

\section{Example 7.}

If a sample of 150 items is tested (or inspected) and there are four failures (or defectives), what is the lower confidence limit at the 0.95 confidence level?

First obtain a $c$ value by subtracting one from the given number of failures, four. The new $c$ value is three. Set the red curve $c=3$ at 0.95 on the diagonal black scale. Read under $n=150$ a lower confidence limit of approximately 0.0095 ; $i$. e., with 95 -percent confidence the true fraction defective is not less than approximately 0.0095 . 
In Example 6, we determined an upper confidence limit at the 0.95 confidence level for $\mathrm{n}=150$ and $c=4$. In Example 7, we determined a lower confidence limit for the same statistics. From Example 6 we are 95 percent confident that $p \leq 0.06$. From Example 7 we are 95 percent confident that $p \geq 0.0095$. Combining the statements we get the 90-percent confidence interval $0.0095 \leq \mathrm{p} \leq 0.06$.

From the foregoing it can be seen that if you want a 90-percent, two-sided confidence interval you can use the computer to obtain a 95-percent upper confidence limit and a 95-percent lower confidence limit. Similarly, if you want a 95-percent, two-sided confience interval you can obtain upper and lower confidence limits at the 97.5-percent level.

\section{Design of the Cumulative Binomial Distribution Computer}

Three sources were used exclusively for obtaining values of the cumulative binomial used in designing the computer. These were:

1. Tables of the Cumulative Binomial Probability Distribution, commonly called the Harvard Binomial Tables (Reference 2). 
2. Tables of the Binomial Distribution Function for Small Values of $p$, (Reference 3 ).

3. A Sandia IBM 1620 computer printout of the cumulative binomial. This printout extended the range of the existing tables.

The Cumulative Binomial Distribution Computer is designed to minimize human errors by the user and great care was taken to accomplish that objective. The contrasting color of the scales, the background color, and the size of the lettering and the scales were chosen to be least confusing to the eye. Comments concerning your experience with this slide rule will be appreciated and may be sent to the Reliability Department, Sandia Corporation, Albuquerque, New Mexico.

On the computer there is a warning that applies to $p$ values in the range of 0.15 to 1.0 : Do Not Use This Range When $n$ Is Less Than 20. The reason is that as $p$ and $n$ approach one, the accuracy of the computer as an approximation of the cumulative binomial decreases. When the computer was being designed two methods were worked out whereby the low range of $n$ with large values of $p$ could be used. However, both of the methods so increased the complexity of the computer that it would have been difficult to use it in any range. Consideration was then given to elimination of the $p$ scale for values greater than 0.15 . This was not done because the range is useful for values of $n$ greater than 20 . It was then found that this range, although not good for approximating the binomial distribution, gives a good approximation of the Poisson 
distribution. The range in which the warning appears may be used if it is kept in mind that the values obtained will, in most cases, be closer to the Poisson than to the binomial.

The side of the slide rule with $n$ values from 100 to 100,000 decreases in accuracy when $c$ approaches $\mathrm{n}$ and $\mathrm{p}$ is greater than 0.5 . The best approximation to the binomial is obtained if, for $p$ values greater than 0.5 , the complement of the $p$ value is used. Since

$$
F(c ; p)=\sum_{x=0}^{c}\left(\begin{array}{l}
n \\
x
\end{array}\right) p^{x}(1-p)^{n-x}=1-\sum_{x=0}^{n-[c+1]}\left(\begin{array}{l}
n \\
x
\end{array}\right)(1-p)^{x} p^{n-x}=1-F(n-[c+1] ;(1-p))
$$

then, if $p$ is greater than 0.5:

instead of $p$ use 1 - p;

instead of c use $n-[c+1]$;

read $F(c)$ remembering that $c$ is $n-[c+1]$ and use $1-F(c)$. 


\section{Example 8.}

The sample size, $n$, is 200 . The fraction defective, $p$, is 0.9 . The acceptance number, $c$, is 174 . What is the probability of acceptance?

Use a $c$ value of $n-[c+1]=200-[174+1]=25$. Use a $p$ value of $1-p=1-0.9=0.1$. Align 0.1 on the $p$ scale with an $n$ of 200 . Where the red curve $c=25$ crosses the diagonal black scale read 0.90 . This is $F(c)$. The probability of acceptance is then 0.10 .

Using the slide rule as explained in the previous example gives the best accuracy for a binomial approximation. However, if the values given in the example ( $n=200, p=0.9, c=174)$ are read directly on the slide rule, a good approximation to a Poisson value is obtained. To generalize, if $p$ values greater than 0.5 are used and $c$ approaches $n$, the values obtained will approximate more closely the Poisson than the binomial.

In summary, the distribution represented by the computer is neither binomial nor Poisson but will approximate either, depending upon the range used. 
1. Muench, J. O. A Confidence-Limit Computer, Sandia Corporation Monograph SCR-159, April 1960.

2. The Staff of the Computation Laboratory, Tables of the Cumulative Binomial Probability Distribution, Harvard University Press (1955).

3. Robertson, W. H. , Tables of the Binomial Distribution Function for Small Values of p, Sandia Corporation Monograph SCR-143, January 1960. 


$$
\text { TID-4500 (34th Edition) }
$$

MATHEMATICS AND COMPUTERS

$\begin{aligned} & \text { No. of } \\ & \text { copies }\end{aligned}$
615

Distribution

Standard Distribution - UC-32 
Issued by

Technical Information Division

Sandia Corporation

Albuquerque, New Mexico 


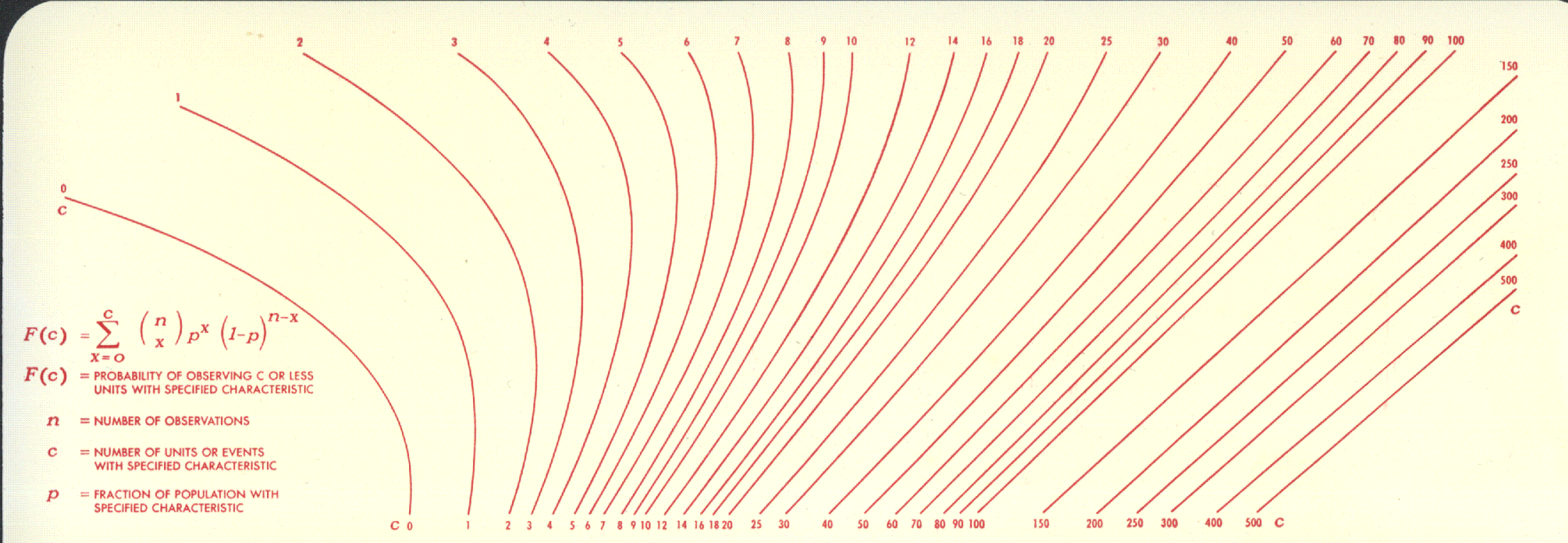











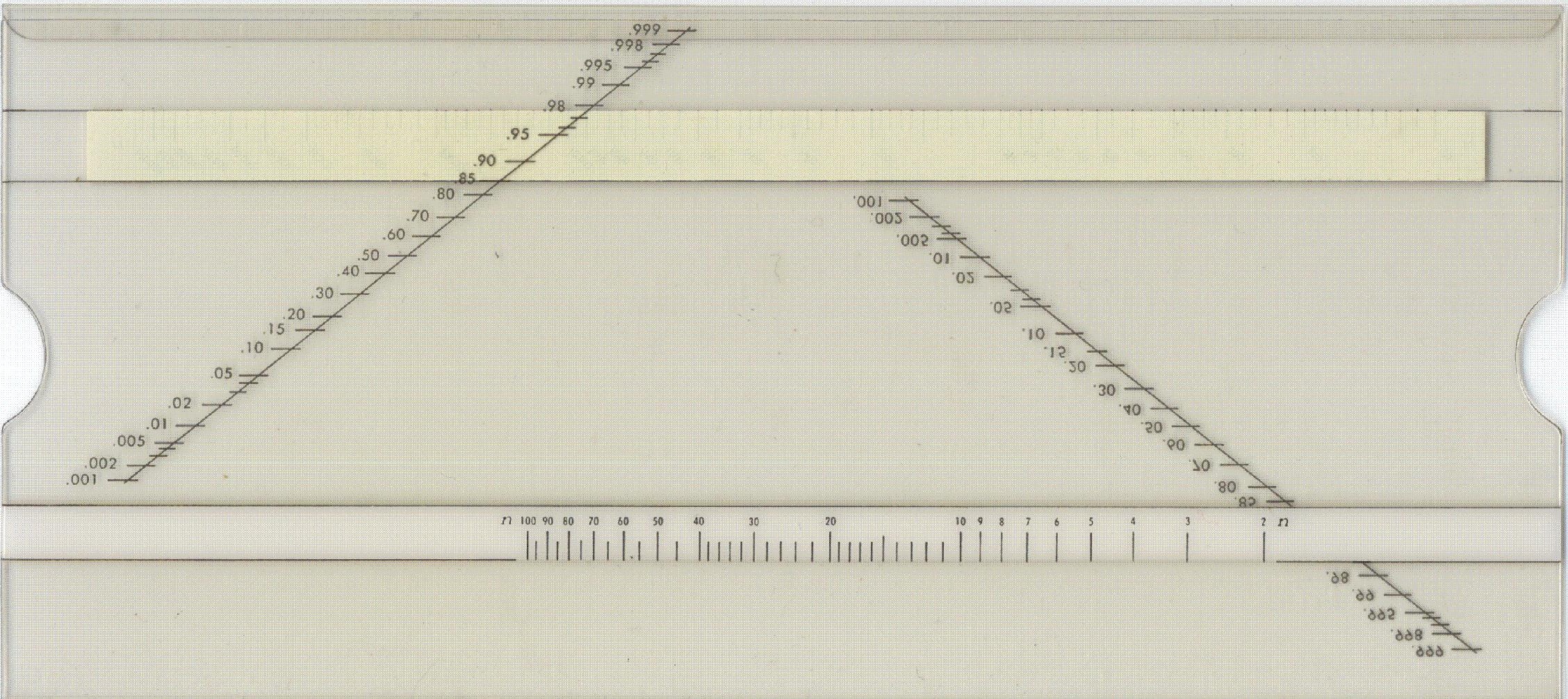




\section{$001-005$}



01

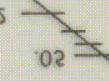

02 政

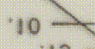

12.

$30>$

20

10

$80 \geq$

$.002+z^{2}$ .0012

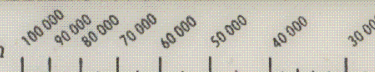





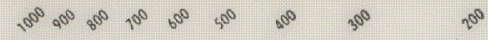

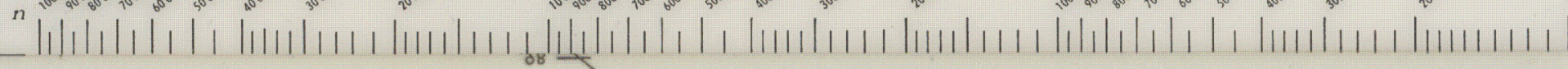

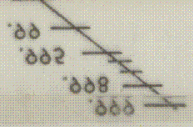






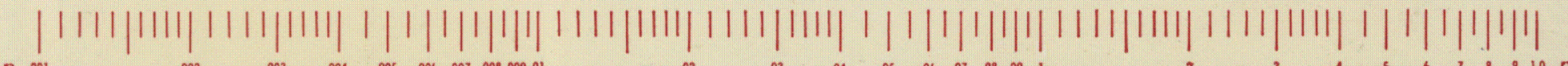

(a) 


\section{INSTRUCTIONS FOR USE}

A. To determine the probability, $\mathbf{F}(\mathbf{c})$, that an event with probability $\mathbf{p}$ occurs $\mathbf{c}$ or fewer times in $\mathbf{n}$ trials:

1. Locate $\mathbf{n}$ on the appropriate face of rule.

2. Move slide to align value of $\mathbf{p}$ with this value of $\mathbf{n}$.

3. Pick red curve for value of $\mathbf{c}$.

4. Read $\mathbf{F}(c)$ from the diagonal black scale where it crosses this red curve.

Example: If $\mathbf{n}=50, \mathbf{p}=.02, \mathbf{c}=2$, then $\mathbf{F}(\mathbf{c})=.92$

B. To determine an upper one-sided confidence limit:

1. Select face of rule which has appropriate value of $\mathbf{n}$.

2. Subtract the desired confidence level from one. Locate this value of $\mathbf{F}(\mathbf{c})$ on the diagonal black scale.

3. Pick red curve for value of $c$.

4. Move slide until this red curve lies at the value of $\mathbf{F}(\mathbf{c})$.

5. Locate value of $\mathbf{n}$.

6. Read opposite the value of $\mathbf{n}$ on the red $\mathbf{p}$ scale the upper confidence limit.

Example: If $\mathbf{n}=50, \mathbf{c}=2$, the confidence level $=.95$, then $\mathbf{p}$, the upper confidence limit $=.12$

C. To determine a lower one-sided confidence limit:

1. Select face of rule which has appropriate value of $\mathbf{n}$.

2. Subtract one from the value of $\mathbf{c}$.

3. Pick the red curve for this new value of $\mathbf{c}$.

4. Locate the desired confidence level, $\mathbf{F}(\mathbf{c})$, on the diagonal black scale.

5. Move the slide until the red curve lies at this value of $\mathbf{F}(\mathbf{c})$.

6. Locate value of $\mathbf{n}$.

7. Read the lower confidence limit opposite this value of $\mathbf{n}$ on the red $\mathbf{p}$ scale.

Example: If $\mathbf{n}=50, \mathbf{c}=2$, the confidence level $=.95$, then p, the lower confidence limit $=.007$

Note:The interval $(.007, .12)$ taken from the examples in $B$ and $C$ is not a .95 two-sided confidence interval but rather a .90 two-sided confidence interval. This is because a probability of .05 (i.e., 1-.95) was allotted to each tail of the distribution giving a total of .10 (i.e., 1-.90) for both tails. (See accompanying booklet.) 